\title{
Die Hirnforschung und der »gefährliche Mensch«. Zur neurowissenschaftlich begründeten Abwesenheit des freien Willens
}

I. Die Hirnforschung rüttelt an einer der Grundsäulen des Strafrechts. Mit der Willensfreiheit soll der Vorwerfbarkeit als zentraler Bestandteil des Schuldstrafrechts der Boden entzogen werden. Neurowissenschaftliche Publikationen ${ }^{1}$, die das Strafrecht insoweit angriffen, evozierten philosophische ${ }^{2}$ und rechtswissenschaftliche ${ }^{3}$ Repliken. Im Windschatten dieser Diskussion aber reproduzieren die Kontrahenten oftmals stillschweigend die Übereinkunft, es gebe Täter, durch das Strafrecht nicht ansprechbar, denen gegenüber allein sichernde oder bessernde, stets aber schuldunabhängige Maßnahmen anwendbar seien. Im Rahmen dieses Abkommens wird der Hirnforschung durchaus ein Wirkungsfeld im Strafrecht zugestanden - zur neurowissenschaftlichen Identifizierung derer, die aus dem Anwendungsbereich des Tatstrafrechts herausfallen. ${ }^{4}$ Die Neurowissenschaften knüpfen insoweit an die binäre Codierung »unzurechnungsfähig/ansprechbar« an. Im Folgenden soll es um die Einsetzung und Selbstplatzierung dieser Codierung vor dem Hintergrund der Willensfreiheit (verstanden als normative Ansprechbarkeit) gehen. Ich werde also versuchen darzulegen, dass die Eröffnung eines erfahrungswissenschaftlichen Wirkungsfeldes innerhalb des auf der Kommunikation normativer Erwartungen fußenden Strafrechts durch eine spezifische historische Form sozialer Kontrolle bedingt ist: die der Willensfreiheit als Erwartung an die Adressaten des Rechts, durch das Recht ansprechbar zu sein und so zu einem

1 Siehe für den deutschsprachigen Bereich etwa: Wolf Singer, DZPhil 2004, S. 235 - 255 sowie ders. DZPhil 2005, S. 707 - 722; Gerhard Roth, DZPhil 2005, S. 691 - 705, ders. DZPhil 2004, S. 223-234 sowie ders. Willensfreiheit, Verantwortlichkeit und Verhaltensautonomie aus der Sicht der Hirnforschung, in: D. Dölling, (Hrsg.) Jus Humanum Grundlagen des Rechts und Strafrechts. Festschrift für Ernst-Joachim Lampe zum 70. Geburtstag, (2003), S. $43-63$.

2 Jürgen Habermas, DZPhil 2004, S. 871 - 890; Wolfgang Detel, DZPhil 2004, S. 891 - 920; Hans-Peter Krüger, DZPhil 2004, S. 257 - 293; Petra Gehring, Philosophische Rundschau 2004, S. 272 - 295; Gesa Lindemann, DZPhil 2005, S. 761 - 781; Otfried Höffe, Der entlarvte Ruck. Was sagt Kant den Hirnforschern? in: Christian Geyer (Hrsg.), Hirnforschung und Willensfreiheit. Zur Deutung der neuesten Experimente (2004), S. 177 - 182; Ansgar Beckermann, Biologie und Freiheit, in: Heinrich Schmidinger, Clemens Sedmak, (Hrsg.) Der Mensch - ein freies Wesen?, (2005), S. $111-124$.

3 Vgl. etwa Thomas Hillenkamp, JZ 2005, S. 313 - 320; Martin Hochhuth, JZ 2005, S. 745 753; Ekkehart Reinelt, NJW 2004, S. 2792 - 2794; Björn Burkhardt, Das Magazin 2/2003, S. 21 - 24; NK-Schild, § 20, Rn. 7; Ernst-Joachim Lampe, ZStW 2006, S. 1 - 43; jeweils mit gewichtigen Unterschieden im Detail. Anders Anja Schiemann, NJW 2004, S. $2056-$ 2059. Kritisch hierzu Reinelt, NJW 2004, S. 2792 sowie Jakobs, ZStW 2005, S. 247 - 266.

4 »Schon differenzierter nehmen sich allerdings Befunde aus, die auf neuroanatomischem und neurophysiologischem Wege bei sog. Vielfach-Gewalttätern erhoben worden sind. Danach sind Defizite in solcher Hinsicht, die schon in Kindheit und Jugend sichtbar werden, bei der Mehrzahl derartiger Delinquenten festgestellt worden.« So Heinz MüllerDietz, GA 2006, S. 340. 
freien Subjekt zu werden. Die kriminologisch und strafrechtlich ausgerichteten Publikationen der Hirnforschung stellen, jedenfalls im Ergebnis und eher weniger denn mehr bewusst, darauf ab, das Scheitern eben dieser Bestimmbarkeit durch die Norm neurowissenschaftlich zu erklären: Der $>$ kalte $<$, psychopathische Täter, der sich durch Strafe nicht abschrecken, sowie die impulsive Gewalt, die ein rationales Kalkül vermissen lasse, sind die beiden Topoi, an denen Neurowissenschaft und Psychiatrie eine Grenze des Tatstrafrechts aufzuzeigen suchen.

Ich möchte die Bedingungen dieses Wissens, welches die Codierung »unzurechnungsfähig/ansprechbar« (und damit auch die Zweiteilung strafrechtlicher Sozialkontrolle in Tat- und Täterstrafrecht, in Schuld und Sicherung) anwendbar macht, aufzeigen. Dazu ist es nötig, zunächst auf die stummen Voraussetzungen strafrechtlicher Theorie und Praxis für die Konstitution des Subjekts des liberalen Strafrechts einzugehen, da erst die >Krise < dieses Subjekts die zweite Seite strafrechtlicher Sozialkontrolle - die kognitive Erwartungssicherung - mitsamt des damit verbundenen ätiologischen, therapeutischen und neurowissenschaftlichen Wissens eröffnet.

II. Im Anschluss an die Arbeiten des britischen Rechtstheoretikers Alan Norrie ${ }^{5}$ verstehe ich das so genannte >criminal justice thinking « als Unterfall des Identitätsdenkens $^{6}$ in der Philosophie. Zeichnet dieses aus, von der falschen Annahme eines fixen, in sich geschlossenen, unvermittelten Subjekts auszugehen ${ }^{7}$, isoliert jenes das Subjekt im Strafrecht von jeglichem sozialen Kontext und reduziert es auf den Zurechnungspunkt eines freien, auf die Beherrschung der inneren Natur angelegten Willens:

»At the heart of such thinking is the idea of a responsible individual subject, who is readily identified in herself and is isolated from the social relations of which she is a part. This is an abstract subject, identified by the generic capacity to be the seat of a will and to act rationally<. It identifies in each individual fixed, general capacities of a psychological kind (to intend, to foresee, to act, to be in control, to be rational). These form the basis for a conception of a fixed subject, separated from other subjects, and identified with the single biological entity that is the human being. On this fixed and

5 Siehe insbesondere dessen Buch Punishment, Responsibility and Justice. A Relational Critique (2000).

6 Für eine genauere Darstellung des philosophischen Identitätsdenkens und der jeweiligen Kritik durch Adorno und Bhaskar siehe Alan Norries Aufsatz: »Bhaskar, Adorno and the Dialectics of Modern Freedom«, in: Journal of Critical Realism, Number 1, 2004, S. 23 48.

7 Norrie bezieht sich insoweit auf Bhaskar (und somit Adorno): entgegen dem Identitätsdenken fordert Bhaskar, in einem dialektischen Abgang von analytischem Denken, mit den üblichen Vorstellungen von Identität zu brechen: »It is to see things existentially constituted, and permeated, by their relations with others; and to see our ordinary notion of identity as an abstraction not only from [its] existentially constitutive processes of formation (geohistories), but also from [its] existentially constitutive inter-activity (internal relatedness)." Roy Bhaskar, Dialectic. The Pulse of Freedom, 1993, S. 125, zitiert nach Alan Norrie, Justice and Relationality, edited version of the presentation at the Critical Realism Day School on Ethics, University of London Union, 19 November 1999, http://www.journalofcriticalrealism.org/archive/ALETHIAv3n1_norrie2.pdf, S. 3. 
separated identity of the subject there depend the various philosophical and legal doctrines which make up ideas of responsibility and blameworthiness." 8

Ersichtlich lässt sich dieses Denken auf die Philosophie der Aufklärung, insbesondere die Immanuel Kants zurückführen. Kant entwickelte mit seiner Sittenlehre ein Programm der sozialen Kontrolle, welches seine Stärke aus der >Autonomie< (d.h. Selbstgesetzgebung) zu gewinnen vermochte, in der die Alternativlosigkeit der reinen Vernunft die Gestalt von Freiheit annehmen konnte: Die Bestimmung durch die reine Vernunft, das allgemeine Gesetz, hat in der Freiheit der Spontaneität zu erfolgen, die Kant bestimmt als Möglichkeit, »eine Reihe von Begebenheiten von selbst anzufangen. ${ }^{9}$ In der Auflösung der dritten Antinomie in der Kritik der reinen Vernunft lässt Kant zwar keinen Zweifel daran, dass jede Handlung des Menschen »vorher bestimmt ist, ehe noch als sie geschieht. $«{ }^{10}$ Dies aber gelte nur für den empirischen Charakter des Menschen. In Ansehung des intelligiblen Charakters aber »gilt kein Vorher, oder Nachher, und jede Handlung, unangesehen des Zeitverhältnisses, darin sie mit anderen in Erscheinung steht, ist die unmittelbare Wirkung des intelligiblen Charakters der reinen Vernunft, welche mithin frei handelt, ohne in der Kette der Naturursachen, durch äußere oder innere, aber in der Zeit nach vorhergehende Gründe, dynamisch bestimmt zu sein [...] « ${ }^{11}$

Für Kant ist Freiheit deshalb versöhnt mit der durchgehenden Kausalität der Phänomena, weil für ihn Freiheit im Handeln gemäß dem vernünftigen Gesetz aufgeht. ${ }^{12}$ In den Grundätzen der Kritik der praktischen Vernunft stellt Kant zwei Aufgaben. Ausgehend davon, dass die bloße gesetzgebende Form der Maximen allein der zureichende Bestimmungsgrund eines Willens sei, müsse nach der Beschaffenheit desjenigen Willens gesucht werden, der durch diese gesetzgebende Form bestimmbar sei.

»Wenn aber auch kein anderer Bestimmungsgrund des Willens für diesen zum Gesetz dienen kann, als bloß jene allgemeine gesetzgebende Form: so muß ein solcher Wille als gänzlich unabhängig von dem Naturgesetz der Erscheinungen, nämlich dem Gesetz der Kausalität [...] gedacht werden. Eine solche Unabhängigkeit aber heißt Freiheit im strengsten, d.i. transzendentalen Verstande. Also ist ein Wille, dem die bloße gesetzgebende Form der Maxime allein zum Gesetze dienen kann, ein freier Wille. $\ll^{13}$

Ist der Wille so als frei vorauszusetzen, sei schließlich das Gesetz zu finden, »welches ihn allein notwendig zu bestimmen tauglich ist.« Dies sei die gesetzgebende Form, »das einzige, was einen Bestimmungsgrund des Willens ausmachen kann. « ${ }^{14}$ Heute wissen wir, dass jene gesetzgebende Form, in der die Willkür des einen mit der

8 Alan Norrie, Justice and Relationality (1999), S. 3. Der kurze, zugängliche Text eignet sich gut für einen Einstieg in das Werk Norries.

9 Kant, Kritik der reinen Vernunft (1974), A 554.

10 Kant, Kritik der reinen Vernunft, A 553.

11 Kant, Kritik der reinen Vernunft, A 553.

12 Das Gesetz der Vernunft bezeichnete Kant als Ursache und sprach folglich von einer »Kausalität der Vernunft in der Bestimmung des Willens«, Kant, Kritik der reinen Vernunft, A 803.

13 Kant, Kritik der praktischen Vernunft, A 52.

14 Kant, Kritik der praktischen Vernunft, A 52. 
Willkür des andern unter einem allgemeinen Gesetz der Freiheit zusammenkommt, nicht der reinen, apriorischen Vernunft entspringt. Geschuldet war und ist dies Gesetz vielmehr der Anforderung an gleichmäßige Beschränkung egoistischer Interessendurchsetzung in der bürgerlichen Gesellschaft, welche in der Philosophie der Aufklärung mit den Weihen des Naturgesetzes ${ }^{15}$ ausgestattet wurde. Diese historische und somit soziale Form ${ }^{16}$, in welche die Verfolgung eigener Interessen somit notwendig gegossen wird, impliziert zugleich bestimmte psychologische Anforderungen an das Funktionieren der Mitglieder der Gesellschaft. Denn dass Freiheit nur dann sei, wenn der Wille sich unmittelbar durch das Gesetz bestimmen lasse, verdeutlicht Kant bei der Behandlung der »Triebfedern« der reinen praktischen Vernunft:

»Das Wesentliche aller Bestimmung des Willens durchs sittliche Gesetz ist: daß er als freier Wille, mithin nicht bloß ohne Mitwirkung aller sinnlichen Antriebe, sondern selbst mit Abweisung aller derselben, und mit Abbruch aller Neigungen, so fern sie jenem Gesetze zuwider sein könnten, bloß durchs Gesetz bestimmt werde. $«{ }^{17}$

Nun stellt sich die Frage, wie im Feld des Phänomenalen (der Motivationen, der Beherrschung der Neigung) reine Vernunft wirksam werden kann. Hierzu muss Kant zwischen dem Dasein und dem Sittengesetz mit der Annahme des intelligiblen Charakters vermitteln. ${ }^{18}$ Jene Vermittlung bedarf des intelligiblen Charakters, der in der Deutung Adornos als Einheit der Person zu fassen sei:

»Hinter den Kulissen des Kantischen Systems wird erwartet, der oberste Begriff der praktischen Philosophie koinzidiere mit dem obersten der theoretischen, dem Ichprinzip, das ebenso theoretisch Einheit stiftet wie praktisch die Triebe bändigt und integriert. Die Einheit der Person ist der Ort der Lehre vom Intelligiblen. « ${ }^{19}$

Als Subjekt der reinen Vernunft solle, so Adorno, die Persönlichkeit die Person als empirisches Einzelwesen unterwerfen. Persönlichkeit sei ein Prinzip jenseits der Einzelperson. Kant wiederhole dabei »die eminent bürgerliche Affirmation der persönlichen Einheit an sich auf Kosten ihres Inhalts, die schließlich unter dem Namen der Persönlichkeit nur den starken Mann übrig ließ. Die formale Leistung der Integration, a priori keineswegs formal sondern inhaltlich, die sedimentierte Beherrschung der inneren Natur, usurpiert den Rang des Guten. ${ }^{20}$

15 Eugen Paschukanis hat darauf verwiesen, dass das Bürgertum in seiner revolutionären Phase naturrechtliches Denken in Anspruch nahm, um dieses gegen feudale Herrschaft in Anschlag zu bringen. Mit der Etablierung bürgerlicher Herrschaft setzte sich der Positivismus in der Rechtsphilosophie durch, damit das Bestehende unhinterfragt bleibe, vgl. Eugen Paschukanis, Allgemeine Rechtslehre und Marxismus. Versuch einer Kritik der juristischen Grundbegriffe (1970 [1924]).

16 Siehe zum Begriff der sozialen Form: Roy Bhaskar, The Possibility of Naturalism (1979), S. 31 - 47. Intentionales Handeln erfolgt danach immer in bereits existierenden sozialen Formen, über die die Handelnde nicht verfügen kann und die, teils unbewusst, jene sozialen Formen reproduzieren. (So die Formen Ehe, Lohnarbeitsverhältnis usw.) Allein hieraus folgt die Unmöglichkeit, soziale Phänomene (wie Kriminalität) biologisch zu erklären.

17 Kant, Kritik der praktischen Vernunft, A 127-129.

18 Theodor W. Adorno, Negative Dialektik (1975), S. 283.

19 Adorno, Negative Dialektik, S. 287/288.

20 Adorno, Negative Dialektik, S. 288/289. 
Bereits nach dieser kurzen Darstellung treffen wir auf die so ins Auge springende Merkwürdigkeit des Kantischen Denkens. Bedarf Freiheit der Spontaneität, darf die freie Handlung zwar nicht durch Zwang oder Angst hervorgerufen werden ${ }^{21}$, muss aber doch durch die Vernunft verlässlich bestimmt sein. Das transzendentale »Ich « muss als Verbindungsglied zwischen den Phänomena und dem Noumenon sich in einer Lücke, einem Unbestimmten bewegen und doch Verlässlichkeit garantieren. Hierin ist das für die moralische Beurteilung einer Handlung konstitutive Nichtwis$\operatorname{sen}^{22}$, die notwendige Abwesenheit und Ausblendung kausal-deterministischer Erklärungen, an deren Stelle die finale oder teleologische Determination durch ein Sollen tritt, zu sehen. So können wir eine Handlung - sei es soziologisch, psychoanalytisch oder eben neurowissenschaftlich - in ein mehr oder weniger enges Netz von Handlungsbedingungen einweben, das für >Freiheit< dann keinen Raum mehr lässt. Andererseits stößt dieses Wissen immer dann an eine Grenze, wenn es den moralischen Vorwurf schwächt. ${ }^{23}$ Es hat dann der Annahme zu weichen, dass das moralisch getadelte Subjekt grundsätzlich für den Anruf der Norm empfänglich ist und somit das Vermögen hat, der Determination durch Handlungsbedingungen die durch Normen wirksam entgegen zu setzen, sich also in Anstrengung des Willens durch die Norm »bestimmen zu lassen.«

Dieses für Freiheit konstitutive >Nichtwissen< erstreckt sich von psychologischen Betrachtungen der Willensfreiheit in der Mitte des neunzehnten Jahrhunderts bis zu gegenwärtigen strafrechtlichen Veröffentlichungen. So verzichtete nach dem Arzt Johann Christian August Heinroth der allmächtige Gott auf die Möglichkeit, menschliches Handeln durchgängig zu bestimmen, um den Menschen, die sich aus Freiheit und nicht aus Angst oder Zwang für das Gute entscheiden, Seligkeit zukommen lassen zu können. ${ }^{24}$ Auch Claus Roxin, der in seinem Lehrbuch auf die Frage der Willensfreiheit eingeht und anführt, die Frage Determinismus/Indeterminismus nicht lösen zu wollen, stellt auf die »unterstellte Möglichkeit« normgemäßen Handelns ab:

»Wenn diese normative Ansprechbarkeit gegeben ist, gehen wir davon aus, ohne dies im Sinne der Willensfreiheit beweisen zu können und zu wollen, dass der Täter

21 So auch Slavojžižek, Parallaxe (2006) S. 27 - 29.

22 So auch Wolfgang Prinz: »Der Willens- und Freiheitsjargon schneidet den Erklärungsregress ab. Er akzeptiert zwar, dass es allerlei Umstände gegeben haben mag, die die Handlungen des Akteurs beeinflusst haben, nicht aber, dass er der Summe dieser Umstände schutzlos ausgeliefert war. Er hätte sich vielmehr auch anders entscheiden können. Deshalb ist er für seine Handlungen verantwortlich. (...) Dies also leistet der Freiheitsjargon für Diskurse und Institutionen der Handlungsregulierung: Er lokalisiert die Quelle von Handlungsentscheidungen in der Person des Akteurs - und damit genau dort, wo Sanktionen und Gratifikationen (auch nachträglich noch) wirksam sind.« Psychologische Rundschau 2004, S. 205.

23 Aus diesem Grund fühlen sich die, die eine Missetat erklären wollen, regelmäßig verpflichtet darauf hinzuweisen, dass damit keinesfalls eine »Rechtfertigung « oder »Billigung « einher gehe.

24 Heinroth, Grundzüge der Criminal-Psychologie; oder: Die Theorie des Bösen in ihrer Anwendung auf die Criminal-Rechtspflege (1833), S. 80. 
auch die Fähigkeit hat, sich normgemäß zu verhalten und sich schuldig macht, wenn er keine der ihm psychisch prinzipiell zugänglichen Verhaltensalternativen ergreift. ${ }^{25}$

Wird dem Täter Freiheit also unterstellt, weil und soweit er als durch Normen ansprechbar gilt ${ }^{26}$, geht eine empirische Dimension - die der Ansprechbarkeit durch die Norm (im Strafrecht: die Einsichts- und Steuerungsfähigkeit) - in eine normative, die der unterstellten Möglichkeit zu rechtmäßigem Verhalten, über: Der Täter macht sich dann schuldig, wenn er eine von der Norm gebotene Verhaltensalternative nicht ergreift; ${ }^{27}$ normativ zugeschrieben wird dabei die aus dem empirischen Befund der Ansprechbarkeit »abgeleitete Möglichkeit zu rechtmäßigem Verhalten. ${ }^{28}$ Bestraft wird nach dieser Konzeption der Täter dann, wenn die Norm nicht in der Handlung wirksam wurde, obwohl sie, der Täter ist ja durch die Norm ansprechbar, hätte wirksam werden können und sollen ${ }^{29}$, gleich, ob sich der Täter in der Tatsituation tatsächlich hätte anders verhalten können. ${ }^{30}$ Mit anderen Worten: Wir brauchen nicht zu wissen, ob sich der Täter tatsächlich hätte anders verhalten können, solange wir ihm normativ (also kontrafaktisch) unterstellen können, von seinen Möglichkeiten keinen Gebrauch gemacht zu haben. So kommt auch Roxin nicht ohne einen blinden Fleck des Nichtwissens (der Unterstellung) in der Herleitung des Schuldvorwurfs aus.

Zwei Punkte seien an dieser Stelle festgehalten: Der moralische (und strafrechtliche) Vorwurf verträgt sich nicht mit einem Wissen um die Ursachen der Handlung. Ein Nichtwissen (das allenfalls durch ein nachvollziehendes »Verstehen« um die Einbettung der Handlung in einen Sinnzusammenhang zu ergänzen ist) ist konstitutiv für den Schuldvorwurf. ${ }^{31}$ Wird einer Handlung abgesprochen, durch Normen bestimmt worden zu sein, tritt die Erklärung durch die (psychiatrische) Krankheit an die Stelle des verstehenden Nachvollzugs normativer Kausalität. Zweitens, und für diesen Zusammenhang entscheidend, impliziert die Übertragungsleistung der Noumena zum Phänomenalen im Kantischen System unausgesprochen eine Einheit eines für die Norm empfänglichen Subjekts. Anknüpfend an Norrie und Adorno kann insoweit von einer morality of form und einer formalen Einheit, die »auf Kosten ihres Inhalts geht«, gesprochen werden.

25 Claus Roxin, Strafrecht Allgemeiner Teil I (2006), § 19 D Rn. 36.

26 Vgl. hierzu nochmals Roxin AT I, § 19 D Rn. 37, der den Täter bei entsprechender normativer Ansprechbarkeit als frei behandelt wissen möchte.

27 Roxin, AT I, § 19 D Rn. 37.

28 »Normativ zugeschrieben wird dagegen [...] die aus diesem Befund abgeleitete Möglichkeit zu rechtmäßigem Verhalten«, Roxin, AT I, § 19 D Rn. 46.

29 Vgl. dazu nur das Beispiel, das Kant in der Kritik der reinen Vernunft, und dort in der Auflösung der Dritten Antinomie, anführt. Der Tadel für eine bösartige Handlung habe seine Grundlage nicht in Natur, sondern Vernunft: »Dieser Tadel gründet sich auf ein Gesetz der Vernunft, wobei man diese als eine Ursache ansieht, welche das Verhalten des Menschen, unangesehen aller genannten empirischen Bedingungen, anders habe bestimmen können und sollen. « Kant, Kritik der reiner Vernunft, A 555.

30 Roxin erklärt seine Freiheitsannahme als von empirischen Gegebenheiten unabhängig, Roxin, AT I, § 19, Rn. 40.

31 Die nationalsozialistische Rechtsphilosophie steht insoweit in hermeneutischer Tradition: Siehe dazu Edmund Mezger, Verstehen als Grundlage der Zurechung (1952) und dort die ebenso erhellende wie erschreckende Falldarstellung. 
III. Wie sehr die Strafrechtswissenschaft in ihrer Antwort auf die Hirnforschung in eben dieser Dichotomie zweier Bestimmungsgründe (Sinn/Bedeutung vs. Körper) verhaftet ist, soll im Folgenden dargestellt worden, bevor ich die Anknüpfung der Veröffentlichungen der Hirnforschung an diese überkommene Zweiteilung behandle.

Subjekt sei, so beispielhaft Ernst-Joachim Lampe in seiner Antwort auf die Hirnforschung, »in den gesetzlichen Straftatbeständen die entscheidungs- und handlungsfreie, sozial durch Normen bestimmbare Person. ${ }^{32}$ Der Täter sei »rechtlich gefordert, rechtmäßig zu handeln, d.h. seine natürlichen Neigungen nötigenfalls den rechtlichen Verpflichtungen unterzuordnen. Diese Forderung ist legitim und muß gestellt werden, weil der Mensch im Laufe der Evolution die Fähigkeit erworben hat, mehr zu sein, als er von Natur aus ist: nämlich zugehörig zu einer bipolaren, nur teilweise kausal, teilweise dagegen teleologisch determinierten Welt. [...] Daher muss das >Vorbild<, welches das Strafrecht als Telos vorgibt [...] zumutbar erreicht werden können. [...] Die empirischen Erkenntnisse, dass die (normale) Person hierzu fähig ist, dass sie die Kraft zur Rechtstreue gewinnen kann, reichen nach meiner Auffassung aus, um in $a b$ stracto mit gutem Gewissen ihre Bestrafung an der Verletzung dieser Pflicht zu orientieren. $\ll^{33}$

Ähnlich fällt Jakobs' Erwiderung auf die Hirnforschung aus. Er stellt ihr gegenüber auf die, an Kants Entgegensetzung von Sinnenwesen und Vernunftwesen angelehnte, Gegenüberstellung von (sittlicher) Person und (biologischem) Individuum ab. ${ }^{34}$ Die Adressaten der »begründeten Zuständigkeiten, die Berechtigten und Verpflichteten, Personen genannt « seien also, soweit normative Erwartungen Orientierung anbieten könnten, »nicht weniger wirklich, als es Individuen mit ihrem kognitiv orientierten Code und ihrem neuronalen Programm oder Psycho-Programm sind. « ${ }^{35}$ Entscheidend sei nicht, ob ein Orientierungszusammenhang sich naturwissenschaftlich darstellen lasse, sondern ob dieser Zusammenhang gesellschaftlich wirklich wird: »Dann leitet $\mathrm{e}[\mathrm{r}]$ die Orientierung. $\ll{ }^{36}$ Ebenso wie bei Lampe ist dieser normativen Orientierung, die sich als Organisationsprinzip nicht naturwissenschaftlich herleiten muss, eine naturwissenschaftliche Grenze gesetzt. Zum naturwissenschaftlichen Teil der gesellschaftlichen Verständigung müsse es ein »Scharnier « geben. ${ }^{37}$ Unmöglich zu erfüllende Pflichten könnten nicht etabliert werden, wobei allerdings Möglichkeiten funktionaler Bewertungen bleiben:

32 Lampe, ZStW 2006, S. 42.

33 Lampe, ZStW 2006, S. 42/43.

34 Jakobs, ZStW 2005, S. 256.

35 Jakobs, ZStW 2005, S. 258.

36 Jakobs, ZStW 2005, S. 258.

37 Bei Jakobs' Gewährsmann Niklas Luhmann heißt es insoweit: »Die genetische Determination des Lebens ist ein unbestrittener Ausgangspunkt. Aber darauf folgt gerade nicht, daß auch Sozialordnungen von da aus determiniert seien (wobei natürlich zu konzedieren ist, daß keine Sozialordnung Bestand haben kann, die verlangen würde, daß die Menschen ständig auf den Händen statt auf den Füßen laufen).« Die Gesellschaft der Gesellschaft (1998), S. 438. 
»Beruft sich die (formelle) Person nach einem Delikt auf ihre individuelle Hirnanatomie, blockt die normativ verfasste Gesellschaft dies in der Regel [...] unter Hinweis auf die Zuständigkeit der Person ab, mit anderen Worten, mit Hinweis auf den freien Willen. $\ll^{38}$

Doch gebe es hiervon Ausnahmen: »Nur verantwortliche Personen, nicht aber kausal-determinierte Individuen, benötigen Freiräume (Kausal-Determinierten sind bequeme Räume angemessen, analog zum Tierschutz). $«{ }^{39}$ Die Rede von der Willensfreiheit habe nun die Aufgabe zu verdeutlichen, dass es im Strafrecht nicht um kausaldeterminierte Individuen, sondern »um die Person, eben die Adresse normativer Erwartungen « gehe. ${ }^{40}$ Willensfreiheit sei also als Metapher dafür zu nehmen, dass der Wille nicht als naturwissenschaftliches, sondern »als normatives Konstrukt die Orientierung leitet. $\ll^{41}$ Weiter schreibt Jakobs: »Nicht anders ist bei Schandtaten zu verfahren: Wenn Irrtum, Krankheit und Zwang nichts erklären, dann ist es die Täterperson, die sich in der Tat zeigt. « ${ }^{42}$ Umgekehrt also kann Krankheit die Tat erklären: Nicht die Freiheit (als Zuständigkeit begründende ungestörte Selbstverwaltung), sondern eine somatische Eigenart der Handelnden erscheint in diesem Fall als Ursprung der Handlung. Nur in diesem Fall trete also eine Entlastungsfunktion ein - die Norm wird nicht als angegriffen gelten. Bei Jakobs muss somit die die Person aufhebende Krankheit vor dem Hintergrund der Annahme jener als Adressatin normativer Erwartungen, als Adressatin der Pflicht zum rechtstreuen Wollen $^{43}$, verstanden werden. Zusammenfassend lässt sich somit anführen, dass nach Jakobs Freiheit als ungestörte Selbstverwaltung der Person nur dann den Hinweis auf die Empirie der Handelnden abschneidet, wenn »Irrtum, Krankheit und Zwang nichts erklären. ${ }^{44}$ Kann Krankheit aber ein Ereignis erfolgreich erklären, greift der mit der Annahme der Freiheit verbundene Erklärungsverzicht nicht.

Entsprechend erklärt sich für Hans Kelsen, auf den ich wegen dessen paradigmatischer Haltung zur Willensfreiheit noch kurz eingehen möchte, der Umstand, dass nur Menschen, nicht aber Tieren und Gegenständen, zugerechnet werde. Nur der menschliche Wille ist für den Neukantianer Kelsen kausal durch Normen bestimmbar. ${ }^{45}$ Eine Zurechnung erfolge somit nur, weil »man annimmt, daß die Vorstellung ihrer Normen nur im Menschen Willensakte verursachen, die wieder das gebotene Verhalten verursachen. Die Erklärung ist also nicht die Freiheit, sondern umgekehrt, die kausale Bestimmbarkeit des menschlichen Willens. ${ }^{46}$ Ausgangspunkt für die Zurechnung sei ein Durchschnittsmensch, der durch Normen bestimmt werden könne. Ein Mensch mit durchschnittlicher Veranlagung der, kausal bestimmt, ein Verhalten an den Tag lege,

38 Jakobs, ZStW 2005, S. 258.

39 Jakobs, ZStW 2005, S. 261. [Herv. im Orig.]

40 Jakobs, ZStW 2005, S. 261.

41 Jakobs, ZStW 2005, S. 264.

42 Jakobs, ZStW 2005, S. 262/263.

43 Jakobs, ZStW 2005, S. 264.

44 Jakobs, ZStW 2005, S. 263.

45 Jakobs bezieht sich zustimmend auf Kelsen (Jakobs NStZ 2005, S 260, Anm. 43).

46 Hans Kelsen, Reine Rechtslehre, 2. Auflage (1960) S. 100. 
welches von der Rechtsordnung verboten sei, sei dieser Rechtsordnung zufolge für eine Handlung und deren Wirkungen verantwortlich. ${ }^{47}$ Wenn er aber »durch gewisse andere als die von der Rechtsordnung vorausgesetzten durchschnittlichen Umstände kausal bestimmt, ein von der Rechtsordnung verbotenes Verhalten an den Tag legt, sagt man, er handle unter unwiderstehlichem Zwang; obgleich der Zwang, unter dem der Mensch unter allen Umständen handelt, stets unwiderstehlich ist.[...] Was man in der juristischen Terminologie als unwiderstehlichen Zwang bezeichnet, ist nur ein besonderer Fall von unwiderstehlichem Zwang, jener nämlich, bei dessen Vorhandensein die Rechtsordnung keine Verantwortung für ein Verhalten vorsieht, für das, wenn es durch andere Ursachen herbeigeführt wird, der so kausal bestimmt handelnde Mensch verantwortlich ist. ${ }^{48}$

Erst die kausale Bestimmbarkeit des Willens macht für Kelsen in einer merkwürdig anmutenden, aber konsequenten Folgerung Zurechnung überhaupt möglich. Mit der Darstellung dieses sozialtechnologischen Verständnisses von Freiheit, das dem strafrechtlichen Denken nahezu durchweg eignet, sind zugleich die Grenzen des Tatstrafrechts aufgezeigt. Um eine Wendung von Jakobs aufzugreifen, die nicht zufällig im Zusammenhang seiner Ausarbeitung des Feindstrafrechts gefallen ist: Kognitive Erwartungssicherung ${ }^{49}$ tritt an die Stelle normativer Erwartungssicherung, wenn die Empfänglichkeit für normative Erwartungssicherung dauerhaft ausgeschlossen ist. So wird der, der in den Worten Jakobs keine ausreichende kognitive Gewähr für seine Rechtstreue zu geben vermag, mittels unmittelbaren Zwangs »eben fremdverwaltet, was heißt, nicht als Person behandelt. $\ll^{50}$ Wo die »ordnungsgemäße Selbstverwaltung der Person « nicht mehr erwartet werde, fehle mit der Vermutung der Rechtstreue auch das Kernelement von orientierungsleitender Personalität und somit die Grundlage freier Selbstverwaltung. ${ }^{51}$ Der Satz: »rechtlich hat jeder Mensch den Anspruch, als Person behandelt zu werden « stellt sich für Jakobs folgerichtig als unvollständig dar. Es »muss zudem festgelegt werden, wer welche Bedingungen der Verwirklichung dieser Personalität herbeizuführen hat. ${ }^{52}$

Nicht schwer fällt, Personalität als das zu identifizieren, was Adorno als naturbeherrschende »Persönlichkeit« bezeichnete, die den intelligiblen Charakter bei Kant ausmache oder als jene morality of form, die Norrie als charakteristisch für das verdinglichte strafrechtliche Denken bestimmte. Entscheidend ist, dass Freiheit als Empfänglichkeit für die Norm (und immer auch: Abschreckung) verstanden ist. So heißt es bereits bei Franz v. Liszt:

$»$ Wer auf Motive in normaler Weise reagiert, ist zurechnungsfähig. Die Zurechnungsfähigkeit entfällt mit jeder Störung des Seelenlebens, die es im Gebiete des Vor-

47 Kelsen, Rechtslehre, S. 101.

48 Kelsen, Rechtslehre, S. 101.

49 Siehe hierzu Alejandro Aponte, Feind oder Bürger? in: Thomas Uwer/Organsiationsbüro (Hrsg.) Bitte bewahren Sie Ruhe. Leben im Feindrechtsstaat (2006), S. 141ff.

50 Jakobs, ZStW 2005, S. 843.

51 Jakobs, ZStW 2005, S. 842.

52 Jakobs, ZStW 2005, S. 843. 
stellens oder des Empfindens oder des Wollens, durch welche die Reaktion anormal, atypisch gestaltet wird « gibt. ${ }^{53}$ Weiter stellt er auf den dadurch gewährleisteten Erfolg im Strafvollzug ab: »Zurechnungsfähigkeit bedeutet demnach die Empfänglichkeit für die durch die Strafe bezweckte Motivsetzung. ${ }^{54}$

Dementsprechend stellte sich auch für Karl Engisch die Frage, warum gegenüber dem Gewohnheitsverbrecher noch ein Schuldvorwurf gemacht werde:

»Die > Vergeltung< durch Strafe hat bei dem Zurechungsunfähigen nach Lage der Dinge keinen Sinn, weil dieser durch die Strafe nicht angesprochen werden kann, während beim Gewohnheitsverbrecher gemäß der Eigenart seiner Persönlichkeit diese Ansprechbarkeit noch gegeben ist. Sollte sie völlig entfallen, so wäre es in der Tat verfehlt, noch mit der Strafe zu arbeiten. Es könnten nur noch Sicherungsmaßnahmen gerechtfertigt sein. $\ll^{55}$

Natürlich begeht Engisch hier zwei Fehler: Weder richtet sich die Gefängnisstrafe primär gegen den Täter, noch ist nachgewiesen, jene könne grundsätzlich »ansprechen« oder gar »bessern«. Doch sollen uns die ungeprüften Voraussetzungen und Ungenauigkeiten ${ }^{56}$ der Verfechter der Sozialverteidigung zunächst nicht weiter beunruhigen. Entscheidend ist der Nachweis, dass und wie die Hirnforschung an jene Zweiteilung der Verbrecher anknüpft und wie diese Dichotomie (in Kategorie und Reaktion) neurobiologisch begründet wird.

IV. Die von mir untersuchten Veröffentlichungen der Hirnforschung ${ }^{57}$ wenden sich teils nicht mehr kategorisch gegen die Annahme des freien Willens, sondern stellen auf die seitens der analytischen Philosophie vorgenommene Bestimmung der Willensfreiheit in einem »weichen « oder kompatibilistischen Sinne ab, um hiervon ausgehend ihr kriminologisches Programm zu rechtfertigen. Für Kompatibilisten sind Entscheidungen »genau dann frei, wenn sie auf Prozessen beruhen, die durch rationale Argumente und Überlegungen beeinflusst werden können. ${ }^{58}$ Bereits dieses Zitat lässt erkennen, dass es hierbei nicht länger um unbedingte Entscheidungsalternativität, sondern um die Beeinflussbarkeit von Entscheidungen durch Bedeutungen und Normen geht. Das kompatibilistische Verständnis von Willensfreiheit geht folglich davon aus, dass auch eine deterministisch geschlossene Welt Raum für Willensfreiheit lässt. In dieser Sichtweise, die auch die weitere Anwendbarkeit moralischer Begriffe gewährleistet, ist eine Handlung frei, wenn der Handelnde anders hätte handeln können,

53 Franz v. Liszt, Strafrechtliche Vorträge und Aufsätze, 2 Bände ([1905] 1970), Aufsätze II, S. 219.

54 v. Liszt, Aufsätze II, S. 220.

55 Karl Engisch, Die Lehre von der Willensfreiheit in der strafrechtsphilosophischen Doktrin der Gegenwart (1963), S. 62.

56 Siehe hierzu bereits G. Th. Kempe: »Franz von Liszt und die Kriminologie«, ZStW 1969, S. $804-824$.

57 Siehe wegen der Einzelheiten: Stefan Krauth, Die Hirnforschung und der gefährliche Mensch. Über die Gefahren einer Neuauflage der biologischen Kriminologie (2008).

58 Ansgar Beckermann, Biologie und Freiheit, in: Heinrich Schmidinger, Clemens Sedmak (Hrsg.), Der Mensch - ein freies Wesen? (2005) S. 118/119. 
sofern er es gewollt hätte. ${ }^{59}$ Im Kern geht es hierbei um die sog. Deliberationsfähigkeit, also das Vermögen, Handlungen durch einen Abwägungsvorgang bestimmen zu lassen. So gesteht auch Gerhard Roth zu, dass aus Sicht der Neurobiologie dieses Vermögen wichtig sei für eine »>vernünftige <, weil langfristige Handlungsplanung. « ${ }^{60}$

Roth, Lück und Strüber greifen in einem in der Deutschen Richterzeitung veröffentlichten Aufsatz das kompatibilistische Verständnis der Willensfreiheit nicht weiter an, sondern stellen die Frage danach, wie mit denjenigen strafrechtlich zu verfahren sei, die eben jenes Vermögen der rationalen Abwägung nicht besäßen.

»Zu bedenken ist allerdings, dass der kompatibilistische Begriff der Willensfreiheit die Fähigkeit voraussetzt, die eigene Motivationslage zu überdenken und auf dieser Grundlage seinem Handeln eine andere, z.B. rechtsgetreue Richtung zu geben, und es erhebt sich die Frage, in welchem Sinne ein Mensch in irgendeinem nachvollziehbaren Sinne schuldig werden kann, der diese Fähigkeit nicht besitzt. $\ll^{61}$

Diejenigen, die die Fähigkeit besitzen, die eigene Motivationslage zu überdenken, können mithin, so der Umkehrschluss, in einem nachvollziehbaren Sinne schuldig werden. Roth, Lück und Strüber kommen in einer jüngeren Veröffentlichung somit auch zu dem Schluss, dass zumindest gegenüber chronischen Gewalttätern auf die Strafe zu verzichten sei:

59 Diese Argumentation geht im Wesentlichen auf Harry Frankfurt zurück. Frankfurt unterscheidet zwischen Wünschen erster und zweiter Stufe. Nach Frankfurt ist eine Person dann frei, wenn ihre Wünsche erster Ordnung und die Wünsche höherer Ordnung übereinstimmen: Der Wille sei dann frei, wenn wir wollen können, was wir wollen wollen. Frei ist eine Person also dann, wenn ihr Handeln durch genau die Wünsche (erster Stufe) bestimmt wird, von der sie auf zweiter Stufe will, dass sie handlungswirksam werden, H. Frankfurt, Journal of Philosophy 1971, S. 5 -20. Eingang in die psychiatrische Literatur findet dieser Ansatz etwa bei Hans-Ludwig Kröber, Die Hirnforschung bleibt hinter dem Begriff strafrechtlicher Verantwortung zurück, in: Christian Geyer (Hrsg.), Hirnforschung und Willensfreiheit. Zur Deutung der neuesten Experimente, 2004, S. 109. Er schreibt: »Wir sind strafrechtlich verantwortlich, wenn wir imstande sind, unsere Entscheidungen von vernünftigen Erwägungen abhängig zu machen $[\ldots] \ll$.

60 Gerhard Roth, Willensfreiheit und Schuldfähigkeit, in: ders, Grün, Klaus-Jürgen (Hrsg.), Das Gehirn und seine Freiheit: Beiträge zur neurowissenschaftlichen Grundlegung der Philosophie, (2006) S. 9 - 27, S. 15.

61 Roth, Lück, Strüber, DRiZ 2005, S. 356. Entsprechend stammen die Beispiele, die von der analytischen Philosophie im Anschluss an Frankfurt herangezogen werden, um Unfreiheit zu illustrieren, durchweg aus Personenkreisen, denen gemeinhin ein Defekt der Selbstbestimmung unterstellt wird: Ein Drogenabhängiger verspüre immer wieder den Drang, die Droge zu nehmen, zugleich wird ihm, der ja mit psychischen, sozialen und medizinischen Defekten ausgestattet ist, der Wunsch unterstellt, seinen Drogenkonsum zu beenden. Nach Frankfurt hat der Drogenabhängige dann einen freien Willen etabliert, wenn sein höherstufiger Wunsch (keine Drogen nehmen zu wollen) dem handlungswirksamen Willen, keine Drogen zu nehmen, Vorrang über sein Bedürfnis (Neigung, Natur), Drogen zu nehmen, verhilft. Warum der höherstufige Wunsch sich bei Abhängigkeit nicht durchzusetzen vermag, wollen aus neurobiologischer Sicht erklären: George Koob, Michel Le Moal, Nature Neuroscience 2005, S. 1442 - 1444; Yavin Shaham, Bruce Hope, Nature Neuroscience 2005, S. 1437 - 1439. Weiterführendes und Kritisches zu dem Ansatz, der einfordert, Freiheit auszuarbeiten, um den Anforderungen an Selbstbestimmung zu genügen, liefert im Hinblick auf Alkohol, Mariana Valverde, Diseases of Will: Alcohol and the Dilemmas of Freedom, (1998). 
»Für das Strafrecht kann all dies nur bedeuten, zumindest bei den chronischen Gewalttätern auf das Prinzip der Strafe als Sühne, das aus dem Begriff der moralischen Schuld erwächst, zu verzichten und sich auf das Prinzip der Abschreckung, der Therapie und des Schutzes der Gesellschaft vor nachweislich nicht therapierbaren Straftätern zu beschränken, wie dies bereits von zahlreichen führenden Strafrechtstheoretikern konzipiert wurde. $\ll^{62}$

Zugleich wenden sich die Autoren und die Autorin aber dagegen, dem Ruf »verhaltensauffällige Jungen und junge Männer präventiv >wegzusperren«« nachzugeben und bezeichnen diese Forderung als »in hohem Maße unethisch. ${ }^{63}$ Der Schutz der Gesellschaft vor »nachweislich nicht therapierbaren Straftätern $«{ }^{64}$ kann freilich der Sache nach nichts anderes sein als das im selben Atemzug gescholtene präventive $>$ Wegsperren $<$ : Ein zentraler Einwand positivistischer Kriminologie an absoluter Strafbegründung besteht darin, dieser Ineffektivität im Schutz vor > gefährlichen Tätern< insofern vorzuhalten, als für diese der Strafzweck Schutz allein über das Nadelöhr der Begehung einer nachweisbaren, rechtswidrigen und schuldhaften Tat möglich sein soll. Ist zudem einmal greifbarer Schutz der Gesellschaft als Zweck staatlichen Strafens anerkannt, bleibt gegenüber sog. »therapieresistenten Tätern« die schuldunabhängige Maßregel der Sicherung das allein Ziel führende Mittel, da diese weder abgeschreckt noch gebessert werden können. ${ }^{65}$ Auch sehen Roth et al. die von ihnen angeführte Dissoziation von Einsichtsfähigkeit und Handlungssteuerung nicht von $§ 20$ StGB erfasst, sondern wollen dessen Anwendungsbereich erweitert wissen:

»Im Folgenden soll es darum gehen, dass zumindest in einigen Bereichen von Straftaten, vornehmlich im Bereich schwerer körperlicher Gewalt (schwere Körperverletzung, Totschlag, Mord) sowie sexuellen Mißbrauchs Einschränkungen kognitiver und emotional-affektiver Leistungen vorliegen, welche die Fähigkeit der Handlungskontrolle stark begrenzen, wobei diese Einschränkungen nicht unter die in $§ 20$ StGB genannten Merkmale fallen. ${ }^{66}$

Ohne also bislang vom Anwendungsbereich des $§ 20$ StGB erfasst zu sein, zeigten »Personen mit erhöhter Aggressivität und Gewaltverbrecher [...] gegenüber der Normalpopulation signifikant häufiger bestimmte strukturelle Hirndefizite im Bereich des Stirnhirns [...] und des Schläfenlappens [...] sowie in limbischen Regionen [...], die alle mit der Entstehung und der Kontrolle affektiver und emotionaler Zustände zu tun haben.«

»Eine Verminderung der Aktivität der Frontalhirns, insbesondere des orbitofrontalen Cortex (OFC), durch Läisionen oder Fehlentwicklungen führt zu einer gesteigerten

62 Roth, Lück, Strüber, NK 2006, S. 58.

63 Roth, Lück, Strüber, NK 2006, S. 58.

64 Roth, Lück, Strüber, NK 2006, S. 58.

65 Vgl. hierzu Roth, Willensfreiheit und Schuldfähigkeit, S. 18, der seine >Theorie< der Strafe in folgende Worte kleidet: »Täter werden danach nicht deshalb bestraft, weil sie >mutwillig < schuldig geworden sind, sondern weil sie von weiteren Taten abgeschreckt oder gebessert werden sollen, falls dies noch möglich ist; anderenfalls muss die Gesellschaft vor ihnen geschützt werden.«

66 Roth, Lück, Strüber, DRiZ 2005, S. 357. 
Impulsivität und >unmoralisch<-kriminellen Verhalten. Personen mit Schädigungen des OFC in frühester Jugend zeigten schwer antisoziales Verhalten auch beim Aufwachsen in normaler Umgebung, sie sind unerziehbar und unbelehrbar. ${ }^{67}$

In der Abschlussbetrachtung weisen Roth, Lück und Strüber dann darauf hin, dass »die Mehrzahl der bisher untersuchten Vielfach-Gewalttäter deutliche neuroanatomische oder neurophysiologische Defizite aufweisen, die in den allermeisten Fällen auch schon in der Jugend sichtbar werden. ${ }^{68}$

Im Hinblick auf die eingangs aufgeworfene Frage nach dem Verhältnis von Willensfreiheit und Schuld ziehen die Verfasser folgenden Schluss:

»In jedem Fall aber handelt es sich bei den Gewaltstraftätern um Faktoren, die meist ihre Wirkung bereits vorgeburtlich, in der Kindheit und frühen Jugend entfalten und nicht der Willensbildung des Straftäters unterliegen. Niemand kann für seine Gene, seine Gehirnentwicklung, für traumatisierende Erlebnisse im Kindesalter und ein negatives soziales Umfeld verantwortlich gemacht werden und damit auch nicht für eine daraus resultierende Neigung zur körperlichen Gewalt. [...] Sofern sich die hier genannten Erkenntnisse weiter erhärten, muss die Schuldfähigkeit chronischer Gewalttäter im Sinne des $§ 20$ StGB überdacht werden. ${ }^{69}$

Bedeutung werde die aktuelle Forschung für die »diagnostischen Möglichkeiten hinsichtlich einer Frühdiagnose« erlangen. Dies erscheine deswegen notwendig, um »normale >jugendliche Raufbolde< von Kindern und Jugendlichen mit einer konstitutionellen Neigung zu körperlicher Gewalt zu unterscheiden - eine Unterscheidung, die heute noch nicht verlässlich möglich ist. ${ }^{70}$ (Folgerichtig unterstreicht Roth die Bedeutung der neurowissenschaftlich fundierten Frühdiagnose für die Entscheidung über die Haftentlassung. ${ }^{71}$ ) Doch ist diese Einschränkung und Zweiteilung der Täter merkwürdig, da aus der Sicht der Hirnforschung schließlich niemand für »seine Gene und seine Gehirnentwicklung « verantwortlich gemacht werden kann. Wieso also soll die Schuldfähigkeit allein gegenüber einem bestimmten Täterkreis überdacht werden, wenn doch streng genommen niemand frei und schuldfähig ist? Nach meiner These knüpft Roth hier an die eingangs erwähnte binäre Codierung an, welche die Grenze des Tatstrafrechts markiert, ohne die Bedingung deren Einsetzung darzulegen. Diese aber ist das Erfordernis, dass das Subjekt durch das Schuldstrafrecht regierbar sei.

67 Roth, Lück, Strüber, DRiZ 2005, S. 357. Roth widerspricht sich dann aber im Folgenden. So würden nicht alle Menschen mit »hirnorganischen oder neurophysiologischen Defiziten« später zu Psychopathen. Ein Drittel der Betroffenen zeige keine oder nur vorübergehende Auffälligkeiten. »Die Gründe hierfür sind unklar und könnten in individuell vorhandenen Reparatur- und Kompensationsfähigkeiten wie auch in einem günstigen Einfluss der Umwelt liegen.« Roth, Willensfreiheit und Schuldfähigkeit, S. 17.

68 Roth, Lück, Strüber, DRiZ 2005, S. 358.

69 Roth, Lück, Strüber, DRiZ 2005, S. 359.

70 Roth, Lück, Strüber, DRiZ 2005, S. 359/360.

71 »Ebenso wird eine entsprechende Diagnosemöglichkeit bei der Entscheidung darüber wichtig sein, ob schwere Gewalttäter erfolgreich therapiert wurden und deshalb aus der Haft entlassen werden können, oder ob sie nur >schauspielern< (wofür Soziopathen berüchtigt sind)«., DZPhil 2004, S. 230. 
Neurowissenschaftliche Experimente stellen insoweit das Wissen bereit, um die durch das Strafrecht nicht Ansprechbaren zu identifizieren.

V. Roth, Lück und Strüber beziehen sich in dem angeführten Aufsatz an zwei entscheidenden Punkten auf ein Experiment von Bechara et al., dessen Ergebnisse 1997 die Zeitschrift »Science « veröffentlichte. ${ }^{72}$ Dieser Aufsatz wird einerseits als »Nachweis« für die Dissoziation von Einsichtsfähigkeit und Handlungssteuerungsfähigkeit herangezogen. ${ }^{73}$ Ferner führten Störungen des orbitofrontalen Cortex »zu einer erhöhten Risikobereitschaft, einer gesteigerten Impulsivität und >unmoralisch<-kriminellem Verhalten (Bechara et al. 1997 [...]) « ${ }^{74}$ Die genauere Betrachtung des Textes lässt aber die damit verfolgte Absicherung jener Behauptungen als prekäre erscheinen. Zunächst soll jedoch das Experiment ${ }^{75}$ skizziert werden.

Bechara und Mitarbeiter ließen ihre Probanden, bestehend aus »normal participiants « und »patients with prefrontal damage«, ein Kartenspiel spielen, »that simulates real-life decision-making in the way it factors uncertainity, rewards, and penalties. ${ }^{76}$ Den Probanden wurden vier Stapel Karten und ein Guthaben von 2000 US-\$ gegeben »and asked to play so that they can lose the least amount of money and win the most.« Das Aufdecken der Karten aus den Stapeln A und B war mit einem Gewinn von 100 US-\$ verbunden, während die Probanden beim Aufdecken von Karten aus den Stapeln $\mathrm{C}$ und D jeweils 50 US-\$ erhielten. Dieser Vorteil erwies sich indes als ein nur scheinbarer, da, für die Teilnehmer nicht vorhersehbar, das Aufdecken der Karten mit einer Strafe in Form von Geldabzug verbunden war, die bei den Stapeln A und B höher ausfiel als bei den Stapeln C und D. Die 16 Probanden mussten also im Laufe des Spiels begreifen bzw. zumindest intuitiv erahnen, dass ein erfolgreiches Spiel erfordert, nur Karten der Stapel C und D aufzudecken, auch wenn sie hierbei langsamer zu Geldzuwachs kamen. Während normale Probanden nach einigen Verlusten begannen, nur noch Karten der risikoärmeren Stapel aufzudecken und die Stapel A und B zu vermeiden, fehlte eine derartige Reaktion bei den Patienten »with prefrontal damage.«Um herauszufinden, ob die Probanden bereits vor oder erst nachdem sie begriffen, dass die Stapel A und B zu riskant waren, korrekt wählten, wurden nach 20 Spielzügen, wobei bereits die ersten Verluste aufgetreten waren, zwei Fragen gestellt: »Tell me all you know about what is going on in this game«, sowie »Tell me how you feel about this game. $\ll{ }^{77}$ Diese Fragen wurden jeweils nach weiteren zehn Spielzügen wiederholt, das Spiel bestand insgesamt aus 100 Zügen. Nach 20 Zügen gaben alle Probanden an, sie hätten keine Ahnung was geschehe. Diese Phase wurde von den Experimentatoren als

72 Antoine Bechara, Hanna Damasio, Daniel Tranel et al, Science 1997, S. 1293 - 1295.

73 »Eine solche Dissoziation konnte in den vergangenen Jahren in der Neuropsychologie eindrucksvoll nachgewiesen werden (Bechara et al., Science 1997)«, Roth, Lück, Strüber, DRiZ 2005, S. 357.

74 Roth, Lück, Strüber, DRiZ 2005, S. 357.

75 Diese experimentelle Anordnung wurde wiederholt von Matthias Brand, Esther Fujiwara, Sabine Borsutzky et al., Neuropsychology 2005, S. $267-277$.

76 Bechara et al, Science 1997, S. 1293.

77 Bechara et al, Science 1997, S. 1293. 
»pre-hunch« bezeichnet, wobei alle »normalen« Probanden nach 50 Zügen angaben, sie hätten eine Vorahnung (»hunch«), dass die Stapel A und B riskanter seien. Demgegenüber drückte keiner der »Patienten« eine derartige Vorahnung aus. Nach etwa 80 Spielzügen konnten sieben von zehn der normalen Teilnehmer schließlich ihre Vorahnung konzeptualisieren, d.h. »expressed knowledge about why, on the long run, decks $\mathrm{A}$ and $\mathrm{B}$ were bad and decks $\mathrm{C}$ and D were good. « Als bemerkenswert bezeichneten Bechara et al., dass auch die drei normalen Teilnehmer, die diese konzeptionelle Phase nicht erreichten, dennoch vorteilhafte Spielzüge durchführten, d.h. sich auch ohne explizites Wissen richtig entschieden. Ebenso bemerkenswert sei, dass die teilnehmenden Patienten, die die konzeptionelle Phase erreichten (»correctly described which were the bad and the good decks «) dennoch unvorteilhaft entschieden:

»Thus, despite an accurate account of the task and of the correct strategy, these patients failed to generate responses and continued to select cards from the bad decks. The patients failed to act according to their correct conceptual knowledge. $\ll^{78}$

Die Autoren bieten folgenden Schluss an: Zwar sei nach verbreiteter Auffassung eine vorteilhafte Entscheidung in komplexen Situationen von offener Abwägung deklarativen Wissens abhängig, doch böten die Experimente auch eine weitergehende Deutung an: »that overt reasoning is preceded by a nonconscious biasing step that uses neural systems other than those that support declarative knowledge. " ${ }^{79}$ Sie schlagen vor, in komplexen Entscheidungssituationen zwei interagierende Prozesse am Werk zu sehen. Neben der offenen oder bewussten Abwägung würden neuronale Systeme aktiviert, »that hold nondeclarative dispositional knowledge related to the individual's previous emotional experience of similar situations. The ventromedial frontal cortices are among the structures that we suspect hold such dispositional knowledge, the activation of which, in turn, activates autonomic and neurotransmitter nuclei (such as those that deliver dopamine to selected cortical and subcortical forebrain regions), among other regions. $\ll^{80}$

Die daraus folgenden unbewussten Signale arbeiteten als verborgene Vorurteile in der Unterstützung kognitiver Evaluation und Abwägung. Diese unbewussten Abläufe eröffneten einen Zugang zum Schatz individueller Erfahrung - im konkreten Fall auf Erfahrungen, die verknüpft sind mit Belohnung und Bestrafung sowie dem jeweils verbundenen emotionalen Zustand. In diesem Licht behinderten, so die Schlussfolgerung, die Verletzungen des orbitofrontalen Cortex den Zugang »to a particular kind of record of previous and related individual experience. $\ll^{81}$

Der Aufsatz ist hinsichtlich der Anordnung des Experiments als auch den gezogenen Schlussfolgerungen angreifbar - hierzu sogleich. Zunächst ist darauf hinzuweisen, dass Bechara et al. die Wörter »kriminell« und »unmoralisches Verhalten« in dem gesamten Text nicht verwandten. ${ }^{82}$ Sie nehmen lediglich an, dass die Probanden mit Schädigungen des Präfrontalkortex bei komplexen Entscheidungssituationen keinen Zugriff auf unbewusst arbeitende Prozesse und ihren Erfahrungsschatz hätten, deshalb in riskanten

78 Bechara et al, Science 1997, S. 1293/1294.

79 Bechara et al, Science 1997, S. 1293.

80 Bechara et al, Science 1997, S. 1294.

81 Bechara et al, Science 1997, S. 1294. 
Situationen ohne Intuition auskommen müssten und teils selbst gegen bessere Einsicht unvorteilhaft handelten. Dabei sind selbst die diesbezüglichen Formulierungen vorsichtig. »The results suggest that, in normal individuals, nonconscious biases guide behavior before conscious knwoledge does. $\ll^{83} »$ We suspect that the autonomic responses we detected are evidence. $\ll^{84}$ Der Verweis auf den Aufsatz von Bechara et al. ist mithin unzutreffend, wenn er als Nachweis für den Zusammenhang von Schädigungen des orbitofrontalen Cortex und kriminellem Verhalten dienen soll. Reduziert man Gewaltkriminalität auf mangelnde Impulskontrolle, liegt es freilich nicht fern, die einmal vorgeblich gefundene neurobiologische Ursache für diese mit der für Gewaltkriminalität identifizieren zu wollen.

Doch sieht sich das dem Aufsatz von Bechara et al. zugrunde liegende Experiment selbst Einwänden ausgesetzt. Die Autoren verfolgen den Anspruch, vermittels eines Kartenspiels »real-life decision-making « zu simulieren, indem dieses die drei Faktoren Unsicherheit, Belohnung und Strafe generiert. Damit wollen sie beweisen, dass Personen mit Schädigungen des Stirnhirns konstitutionell, also aufgrund dieser Schädigungen, im Experiment schlechter abschneiden als normale Versuchspersonen. Selbst die begriffliche Durchdringung der experimentellen Anordnung kann ihnen nicht helfen, das Spiel ebenso erfolgreich zu bewältigen wie die gesunden Konkurrentinnen. Da die zu vollziehenden Entscheidungen die des wirklichen Lebens simulierten, ist zugleich die eingeschränkte Lebenstauglichkeit der nicht normalen Probanden bewiesen. Das Experiment bestimmt, ohne Möglichkeit dies zu ändern, die allein richtige Entscheidung im Hinblick der ihm immanenten Zielvorgabe »to play so that they can lose the least amount of money and win the the most. ${ }^{85}$ Die experimentelle Anordnung ist eine geschlossene, da sie dem Probanden in der Erwartung einer richtigen Wahl gegenübertritt. ${ }^{86}$ Dabei wird davon abgesehen, dass im Alltagsverständnis das Kartenspiel um Geld weder primär noch ausschließlich der Erzielung eines finanziellen Gewinns dient. Gehen Teilnehmer mit der Erwartung an ein Kartenspiel, sich riskanten Situationen auszuset-

82 Wie bereits angeführt, sprechen Roth, Lück und Strüber davon, dass Schädigungen des orbitofrtontalen Cortex »zu einer erhöhten Risikobereitschaft, einer gesteigerten Impulsivität und >unmoralisch`-kriminellem Verhalten (Bechara et al., Science 1997 [...])« führten, Roth, Lück, Strüber, DRiZ, 2005, S. 357.

83 Bechara et al, Science 1997, S. 1293.

84 Bechara et al, Science 1997, S. 1294. [Hervor. durch den Verf.]

85 Bechara et al, Science 1997, S. 1293.

86 Zur Differenz zwischen »offener « und »geschlossener Frage« unter Bezugnahme auf Plessner siehe Lindemann, DZPhil 2005, S. 767: »Wenn das Verfahren der Konstruktion der Erkenntnisrelationen am Prinzip der geschlossenen Frage orientiert wird, gestaltet sich der Zugang zum Gegenstand folgendermaßen: In die Frage geht ein Problementwurf ein, der einen Vorentwurf des Gegenstandes enthält. Durch diesen wird konstitutiv festgelegt, was als ein zu untersuchender Gegenstand erscheinen und wie er auf die Forschungsfrage antworten kann. «Es soll andererseits nicht bestritten werden, dass die der Fragestellung zu Grunde liegende Rationalität unter den bestehenden Umständen »wirklich« ist. So kommt den Menschen angesichts der Konkurrenz keine substantielle Handlungsalternative zu: Entweder folgt man den Spielregeln des Marktes - oder man geht unter. Eine Kritik dieser Fragestellung kann also nicht allein darauf abzielen, dass das durch die experimentelle Fragestellung erst hervorgebrachte Wissen »sozial konstruiert« sei, sondern muss auf den affirmativen Gehalt des im Experiment Reproduzierten abstellen. 
zen, mithin >Nervenkitzel< mit einem etwaigen Verlust zu erkaufen, mag >unvernünftig < riskantes Verhalten also durchaus von der individuellen Zwecksetzung gedeckt sein. Erst die geschlossene Anordnung des Experiments zwingt die Probanden in die durch das Experiment gesetzte Rationalität. Schließlich ist der Schluss von dem schlechten Abschneiden bei dem Test auf analoge Schwierigkeiten in alltäglichen komplexen Entscheidungssituationen unzulässig. Konfrontiert mit Entscheidungen auf unsicherer Tatsachenbasis und der Aussicht auf Nachteile, können die Betroffenen immer noch Alternativstrategien entwickeln, um vorhandene Defizite auszugleichen. Der entscheidende Einwand aber ist, dass eine dem Alltagsverständnis entnommene Anforderung an Rationalität herangezogen wird, um diese auf dem Umweg über ein naturwissenschaftliches Experiment mit den höheren Weihen der Zwangsläufigkeit auszustatten. So ist für Bechara die normative Erwartung an den zurückhaltend-abwartenden Entscheidungsmodus des Bürgers das a priori der experimentellen Fragestellung. Zugleich löscht dieses Experiment den alltäglichen Weltbezug des Subjekts aus, das als Versuchsperson nur reduziert und abstrakt wahrgenommen wird. Dessen »Erfahrungs- und Handlungsmöglichkeit in der realen Welt « werden somit gleichsam »gekappt. $\ll{ }^{87}$ Dies ist nicht in erster Linie eine forschungsethische Frage, sondern verweist auf die Unmöglichkeit der bruchlosen Übertragung von Befunden, die in geschlossenen Systemen (Experimenten) gewonnen werden (und nur dort gelten), auf offene Systeme, mithin die Gesellschaft. ${ }^{88}$

VI. Nicht auf alle Einwände naturwissenschaftlicher Seite gegenüber dem Schuldstrafrecht kann hier angemessen eingegangen werden. In einer Abschlussbetrachtung sollen allein einige Kernthesen zusammengefasst werden: Die Hirnforschung verzichtet auf einen generellen (und nicht Erfolg versprechenden) Angriff auf das Strafrecht und bietet ihre Hilfestellung für die Identifizierung einer Risikopopulation an. Dass aus dem neurowissenschaftlichen Experiment keine Gesetzmäßigkeiten gezogen werden können, ist insofern unschädlich, als die Rechtsprechung für die schuldunabhängigen Maßnahmen der Besserung und Sicherung allein Prognoseinstrumentarien einfordert, die Gefährlichkeit ausreichend zu belegen haben. ${ }^{89}$ Die neurowissenschaftliche Problematisierung erfolgt entlang zweier Topoi: der impulsiven, irrationalen Gewalt

87 Klaus Holzkamp, Manuskripte zum Arbeitsprojekt »Lebensführung«. In: Forum Kritische Psychologie 36 (1996), S. 18.

88 Siehe zu den Einzelheiten: Roy Bhaskar, The Possibilitiy of Naturalism (1979), Kaptitel 1, S. 1- 30.

89 Das BVerfG begründete im Jahr 2004 die Verfassungsmäßigkeit der Sicherungsverwahrung nicht zuletzt mit der zweifelhaften Annahme gesteigerter Zuverlässigkeit von Prognosegutachten: »Die Prognose ist und bleibt als Grundlage jeder Gefahrenabwehr unverzichtbar, mag sie auch im Einzelfall unzulänglich sein. In der Praxis der forensischen Psychiatrie hat sich im Übrigen das Wissen um die Risikofaktoren in den letzten Jahren erheblich verbessert, so dass über einen Teil der Delinquenten relativ gute und zuverlässige prognostische Aussagen gemacht werden können.«, BVerfG, 2 BvR 2029/01 vom 5. Februar 2004, Absatz-Nr. 101 102, http://www.bverfg.de/entscheidungen/rs20040205_2bvr 202901.html. In anderen Worten: die Prognose ist notwendig, selbst wenn sie unbrauchbar ist. Siehe zu dieser Form der >Gerechtigkeit grundlegend: Malcolm Feeley, Jonathan Simon, Actuarial Justice: the Emerging New Criminal Law, in: David Nelken, (Hrsg.), The Futures of Criminology (1994) S. 173 - 201. 
einerseits und der kalten, zweckgerichteten Gewalt des >Psychopathen< andererseits. Jene wird mit dem Spiegel des Botenstoffs Serotonin im Gehirn erklärt, während diese $\gg$ neurobiologisch noch nicht genügend verstanden ist $\aleph^{90}$, aber ebenso in einem naturwissenschaftlichen Beschreibungssystem gefasst werden soll. Die planlose Impulsivität, die die Konsequenzen der Handlung nicht antizipiere, beschäftigt die Hirnforschung, da gegenüber impulsiven, nicht vorausschauenden Individuen die Rationalität des Strafrechtssystems nur eingeschränkt zur Geltung kommen könne: »Because the rewards of violent behavior usually precede its penalties, time discounting can be seen as a mechanism underlying nonplaning impulsive violence. ${ }^{91}$ Hinsichtlich der kalten, berechnenden Gewalt stellen Hirnforscher den Zusammenhang zwischen der Abschreckungsfähigkeit durch die Generierung von Angst und dem Hippocampus wie folgt dar: kalte Gewalttäter »display highly significant negative correlations with the posterior half of the hippocampus and the degree of psychopathy. $\ll^{92}$ Dabei wird die Brücke zum Zusammenhang von Konditionierungsfähigkeit und Neurobiologie geschlagen. Unter Bezugnahme auf Tierversuche legen die Autoren dar, dass Verletzungen, die bestimmte Neuronen des Hippocampus zerstörten, »lead to impairment in conditioning to contextual fear. ${ }^{93}{ }^{93}$ Übertragen auf Psychopathen führen die Autoren zum Scheitern von Konditionalisierung über Angst bei Psychopathen aus:

»Similar deficits have been observed in psychopathic subjects. Psychopaths have been repeatedly shown to have abnormal or absent neurophysiological responses compared with controls [...] in studies of aversive conditioning. For instance, older studies, which used electric shock as an aversive unconditioned stimulus, suggested that psychopaths fail to experience fear in anticipation of the shock, i.e. deficit in fear conditioning, as measured by changes in skin conductance. These findings promoted a hypothesis suggesting that psychopaths are impoverished in >conditionability.< They fail to learn and to profit from experience, because they do not generate sufficient arousal, fear or anxiety necessary for associative learning. $\ll^{94}$

Im Kern geht es also in beiden Fällen um die Nichtregierbarkeit durch das Strafrecht ${ }^{95}$, die durch Impulsivität (d.h. mangelndes Absehen der künftigen Folgen) sowie mangelnde Konditionierung über Angst (Psychopathie) entsteht. Ist damit die Abhängigkeit der neurowissenschaftlichen Wissensproduktion von einem notwendigerweise verdinglichten ${ }^{96}$ und erst somit regierbaren Subjekt aufgezeigt, stellt sich zugleich die Frage nach der bio-politischen Tragweite der Hirnforschung. Denn diese stellt ein Wissen um die biologische Tauglichkeit eines Teils der Bevölkerung bereit, der aufgrund dieses Wissens disqualifiziert und vom Schuldstrafrecht ausgeschlossen werden kann. ${ }^{97}$ Wie sehr die Sorge um die biologische Brauchbarkeit der Bevölkerung heute

90 Roth, Lück, Strüber, Psychobiologische Grundlagen aggressiven und gewalttätigen Verhaltens (2005), S. 88.

91 Jan Volavka, Neurobiology of Violence (2002), S. 191.

92 Laakso et al, Behavioural Brain Research 2001, S. 190.

93 Laakso et al, Behavioural Brain Research 2001, S. 191.

94 Laakso et al, Behavioural Brain Research 2001, S. 191.

95 Siehe hierzu Michel Foucault, Geschichte der Gouvernementalität II. Die Geburt der Biopolitik (2004), S. 346ff. 
noch die psychiatrische Fragestellung konturiert, beweist folgendes Zitat zweier Psychiater:

»Impulsivity, aggressiveness, and associated aggressive behaviour are common traits, the expression of which must be carefully modulated to ensure the success of individuals, small groups, and large societies, especially with the current framework of rapid globalization. ${ }^{98}$

Ich möchte nun zum Schluss kommen und vorschlagen, der kriminologischen Hirnforschung politisch zu begegnen. Damit ist gemeint, soziale und politische Voraussetzungen dieser Forschung, die allzu gerne unterschlagen werden, aufzuzeigen und in Frage zu stellen. Hierzu zählt, das dem Strafrecht zu Grunde Liegende und Unterworfene, d.h. dessen Subjekt, dialektisch (relational) auf die Bedingungen seiner Entstehung in der bürgerlichen Gesellschaft zurückzuführen: Erwächst dieses Subjekt aus einer politischen Form, ist dessen Scheitern eine politische und keine neurobiologische Frage. Das Subjekt des Strafrechts als politische Form zu begreifen bedeutet, diese als historisch und grundsätzlich änderbar zu verstehen. Demgegenüber nimmt die naturwissenschaftliche Erklärung abweichenden Verhaltens die Gestalt politischer Herrschaft an, da dieses Wissen allein aufgrund seiner wissenschaftlichen Form zu Handlungen aufruft, seine Herkunft aus der politischen Form >Strafrecht $<$ dabei aber verleugnet. Der liberale Individualismus vermag gesellschaftliche Herrschaft in der Freiheitslehre insoweit zu verschleiern, als das Scheitern von Normumsetzung moralisch bis biologisch/psychopathologisch gewendet und an einem Individuum festgemacht wird. Sowohl der moralische Tadel als auch die (vorgeblich gelungene) neurobiologische, exkulpierende Identifizierung der Grundlagen abweichenden Verhaltens täuschen darüber hinweg, dass beide Formen der >Zurechnung ‘ gesellschaftliche Komplexität funktional reduzieren und von der antagonistischen Gesellschaft als Entstehungsbedingung enttäuschter Norm, Tat und Täter absehen. Betrachtet man die Freiheitslehre in diesem Sinne als abstrakt und repressiv, wäre Freiheit die Freiheit vom Zwang, einen regierbaren Willen haben zu müssen: »Vielleicht wären freie Menschen auch vom Willen befreit. ${ }^{99}$

96 Alan Norrie weist darauf hin, dass die von ihm kritisierte morality of form eine historisch notwendige Trennung des Subjekts vom Sozialen sei, um Verantwortung zuschreiben zu können. Vgl. Norrie, Justice and Relationality, S. 4: »If we want to pick out individuals and say that they are to blame for wrongs in a situation where wrongs are plainly social phenomena with structural causes, an analytical inscription and circumscription of the individual has to be achieved. Picking out the person and separating her from her context, for which she is largely not responsible, is a necessary requirement of a system that wants to blame individuals.«

97 Siehe hierzu: Stefan Krauth, Die Hirnforschung und der gefährliche Mensch (2008).

98 Lesch, Merschdorf, Behavioral Sciences and the Law 2000, S. 597.

99 Adorno, Negative Dialektik, S. 261. 